\title{
Some Equations for the External Space and Their Implications on the Inverse Cone Concept and the Geometry of the Physical Universe
}

\author{
J. G. Lartigue \\ Retired Professor, National University of Mexico, Mexico City, Mexico \\ Email: jmlg5@hotmail.com
}

How to cite this paper: Lartigue, J.G. (2020) Some Equations for the External Space and Their Implications on the Inverse Cone Concept and the Geometry of the Physical Universe. Journal of Modern Physics, 11, 1005-1012.

https://doi.org/10.4236/jmp.2020.117063

Received: May 6, 2020

Accepted: July 7, 2020

Published: July 10, 2020

Copyright $\odot 2020$ by author(s) and Scientific Research Publishing Inc. This work is licensed under the Creative Commons Attribution International License (CC BY 4.0).

http://creativecommons.org/licenses/by/4.0/

\begin{abstract}
In previous works, they were proposed a photonic model of the Big Bang [1] and several parameters derived from the Hubble-Lemaitre equation [2]. Since these parameters result higher than the classical ones and, otherwise, the General Theory of Relativity does not apply far away the Physical Universe, in this paper, it will be revised the adequacy of such parameters in the external Space and their influence on the relativistic concept of the cone of time. As well, it will be intended to define the Physical Universe geometry accordingly to a thermo-dynamical analysis of the Big Bang.
\end{abstract}

\section{Keywords}

De Sitter Equation, The Inverse Time Cone, Big Bang Characteristics

\section{Background}

From to the Schwarzchild rule, the Luminosity of the Physical Universe should have been $\mathfrak{S}_{\mathrm{pu}} \sim 1.8 \times 10^{59}(\mathrm{erg} / \mathrm{s})$ which, multiplied by the Big Bang duration, $10^{12}(\mathrm{~s})$ [3], gives an energy production $\sim 2 \times 10^{71}(\mathrm{erg})$. However, the energy of to the original $\mathrm{CMB}$ radiation would be much higher: the present density of the $\mathrm{CMB}$ relic is about 416 (photons $/ \mathrm{cm}^{3}$ ) [4]; applying the cosmological principle, it is obtained a total number of CMB photons, in the total Universe volume $(3 \times$ $10^{85} \mathrm{~cm}^{3}$ ), of $1.2 \times 10^{88}$ (photons) with a heat content of $3 \times 10^{88}$ (erg). By assuming that the final temperature of the Big Bang period was $\sim 2 \times 10^{4}(\mathrm{~K})$, that would mean a $z$ factor about $2 \times 10^{3}$ which implies an original energy of the $\mathrm{CMB} \sim 6 \times 10^{91}$ (erg). Therefore, the fraction of the total Big Bang energy devoted to the creation of the Physical Universe was $\sim 10^{-20}$ and the Luminosity corresponding to the total Universe age would now be: $\mathfrak{L}_{\mathfrak{u}} \sim 1.3 \times 10^{74}(\mathrm{erg} / \mathrm{s})$. 
The Einstein's equation of General Relativity was modified by De Sitter for a powder cloud as:

$$
\boldsymbol{R}^{i j}-\left(\frac{1}{2}\right) g^{i j} \boldsymbol{R}=8 \pi G \rho \boldsymbol{v}^{i j}+\Lambda g^{i j}
$$

Since $\rho=0$ in the external Space and $\Lambda$ was discarded by Einstein, the volumetric accelerations terms would cancel out. Otherwise, though the gravitational intensity does not vanish at distances even higher than $\boldsymbol{r}_{o}$, the expansion velocity of the vacuum Space is not a function of the $\boldsymbol{g}$ value; it depends on the universal constant acceleration $\left(\Gamma_{H}\right)$ and time.

Therefore, it could be useful to summarize some equations that may be applied to describe the external Space dynamics. Those are:

- The Hubble parameter:

$$
H=2 / t\left(\mathrm{~s}^{-1}\right)
$$

- The Hubble velocity:

$$
\boldsymbol{v}_{H}=H \cdot \boldsymbol{r}=2 \boldsymbol{r} / t(\mathrm{~cm} / \mathrm{s}) .
$$

- The constant Hubble acceleration:

$$
\Gamma_{H}=H^{2} \boldsymbol{r} / 2\left(\mathrm{~cm} / \mathrm{s}^{2}\right) \text {. }
$$

- The Space expansion velocity:

$$
\boldsymbol{v}_{s}=\Gamma_{H} \cdot t(\mathrm{~cm} / \mathrm{s}) .
$$

- The Hubble field potential:

$$
V_{H}=H^{2} \boldsymbol{r}^{2} / 4\left(\mathrm{~cm}^{2} / \mathrm{s}^{2}\right) \text {. }
$$

Simultaneously, it has been assumed [2] that the Big Bang was a singularity, i.e. the most probable origin of time, Space, matter and the physical laws. So, the Space started immediately an expansion, a fact discovered by Hubble-Lemaitre in 1929 [5] and confirmed as an accelerated movement by Riess et al. [6]. The author assumed the simplest case: that of a constant acceleration, as a function of distance and time. Besides it was proposed an equation for $\mathrm{y}$ to avoid an imaginary proper time if $\boldsymbol{v}>\boldsymbol{c}$, [2]. However, the possibility of an imaginary time has been mentioned by Hawking [7] as a component of the Euclidic Space, adequate to describe the expansion velocity of the Space.

The Poisson equation for the Hubble potential was obtained in reference [1] as

$$
\nabla^{2} V_{H}=H^{2}\left(\mathrm{~s}^{-2}\right),
$$

which permits to express the scalar of curvature of the free Space as:

$$
R=6 H^{2}\left(\mathrm{~s}^{-2}\right) \text {. }
$$

The Einstein Equation (1) as a function of a Hubble tensor is:

$$
G_{\mu v}=g_{\mu v} 3 H^{2} \text {. }
$$

The scalar of this tensor would be: 


$$
T^{H}=3 H^{2}=\Lambda .
$$

\section{The Variation of the Cone of Time}

In a previous work [8] it was concluded that, in the Physical Universe, the light velocity does not depend on the gravitational potential, i.e. it is a universal constant. Besides, since the Space expands at growing velocities (including the matter of the Hubble Flux) it is very probable that the light accelerates simultaneously as the Space does; this would imply an apparent dilemma: if the Space expansion work is carried out by only the Hubble potential $\left(V_{H}\right)$, without any change in the photon's energy or, opposed, the photon's work implies a redshift: $W=h \cdot \Delta v$ that would limit the photon's life-time. In the author's opinion all works are done at expenses of $V_{H}$, without any wear of the expanding matter.

The Special Relativity concept of the inverted cone of time is based on the principle of the light speed constancy; so, the radius of the cone should be calculated by the straight line equation: $\boldsymbol{r}=\boldsymbol{c} \cdot t(10)$. If the light would travel in the external Space, at the same velocity than Space does, the distance should be determined by the equation:

$$
\boldsymbol{r}=\frac{\Gamma_{H}}{2} \cdot t^{2}
$$

Substitution of the acceleration constant drives to the equation:

$$
\boldsymbol{r}=10^{-7} t^{2}
$$

i.e. the cone slant follows a parabolic trajectory respect to time, as shown in Figure 1. In the interval from $t=0$ to $t=10.5 \mathrm{~Gy}$, the radius of the straight cone basis was higher than the parabolic one though, after such a time, the parabolic

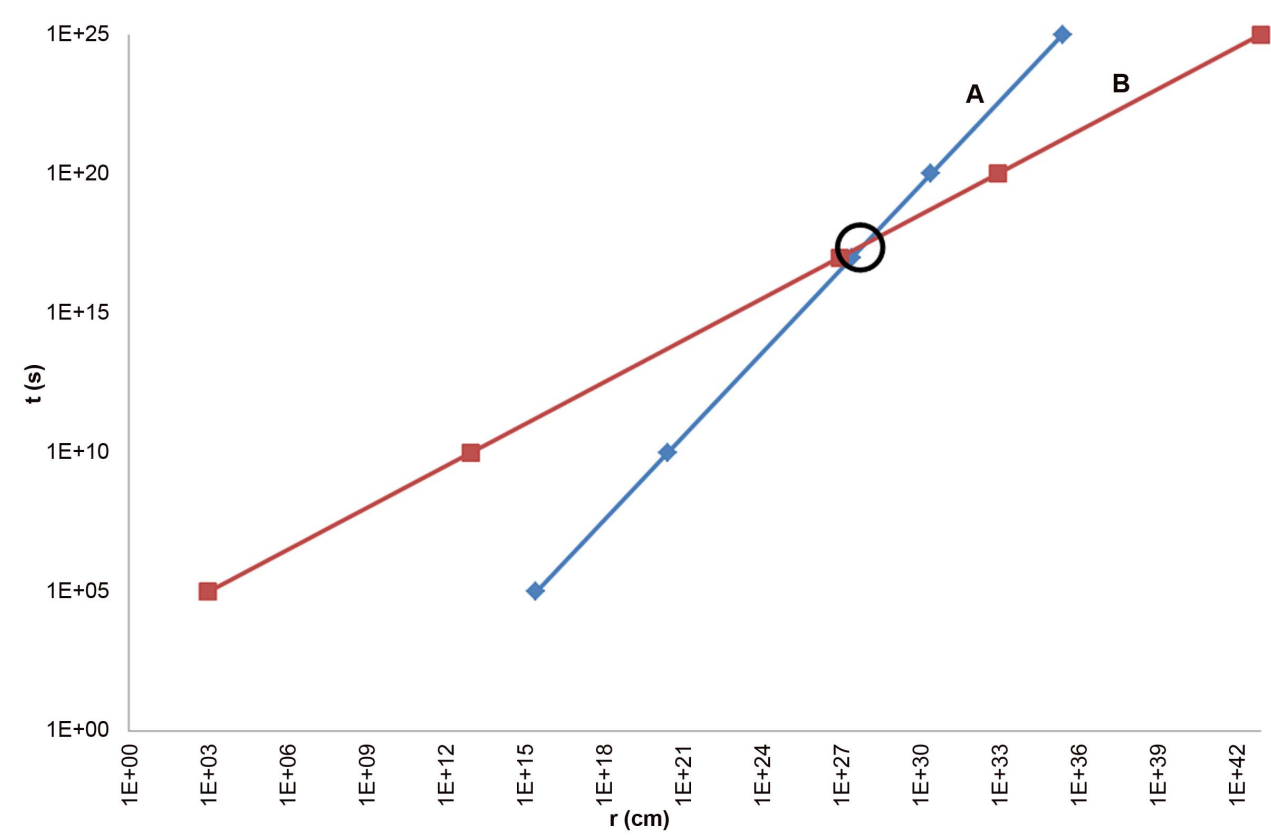

Figure 1. The slants of the straight (A) and the parabolic (B) cones of the Space Expansion. Both lines intersect at an Universe age of $\sim 10^{10}(\mathrm{y})$. 
cone radius grows faster than the straight one. Even so, at any future Space velocity, the so-called "elsewhere zones" will never disappear.

\section{The Lorentz Transform in an Accelerating Space}

The basic equation of the Lorentz transform is:

$$
\boldsymbol{v}_{x}^{\prime}=\boldsymbol{v}_{x}-\boldsymbol{v} ;
$$

$v$ is the separation velocity of two inertial frames of reference (primed and not-primed) and the sub index $\mathrm{x}$ refers to the same coordinate.

The Space accelerated expansion does not correspond to an inertial frame, though in the Physical Universe they may be present both types of frames. So, it could be proposed the following equation:

$$
v_{x}=v_{x}^{\prime}+\Gamma \cdot t
$$

where: $\boldsymbol{v}_{x}=$ velocity of matter in the Space,

$\boldsymbol{v}_{x}^{\prime}=$ velocity of matter in the Physical Universe

$\Gamma \cdot t=$ expansion velocity of Space at time $t$. Each one of the application points of this vector corresponds to a co-moving coordinate.

Since Equation (14) is a vector sum, the velocity of matter $\boldsymbol{v}_{x}$ could be lower or higher than that of the Space. Given the order of magnitude of such velocities, it is probable that the matter velocity $\boldsymbol{v}_{x}^{\prime}$ would always be considered as a peculiar velocity.

\section{The Universe Form}

Since the Space expansion occurred in all directions around the Big Bang point (same as it did the Physical Universe) and if this one is composed of the known matter, all of that must now be confined into a ring: a spherical shell geometry, as a spherical tokamak. The nice diagrams of the Universe development, such as that of reference [6], show the History of the Universe expansion into an angle of $<60^{\circ}$. If it would be extended to a $4 \pi$ geometry, it could be confirmed that all of the surviving matter must now be confined into a spherical shell. It means that the condensation of radiation would have started when the Universe temperature must be lower than $2 \times 10^{4}(\mathrm{~K})$, i.e. at the end of the Big Bang. So, as shown in the Universe cross section of Figure 2, if $\boldsymbol{r}_{B}$ was the Big Bang radius, the two radii $\left(\boldsymbol{r}_{i}\right.$ and $\left.r_{e}\right)$ correspond to the width of the Physical Universe; then, $\boldsymbol{r}_{\mathrm{i}}$ would be the radius of the past expanding Space. Consequently a complete light turn, lengthways the annular Physical Universe, would take a time $t_{p u} \sim 13 t_{o}$.

The condensation of photons to leptons could not be possible at the initial Big Bang temperature of $10^{31}(\mathrm{~K})$, i.e. much higher than the binding energies of leptons $(\sim 1 \mathrm{eV})$. Therefore such a condensation would have started at the end of the Big Bang period ( $t_{B} \sim 32,000$ years) [3]. The necessity of photonic condensation opposes to the common theory of a short Big Bang (with small dimensions and a high temperature) which required to be followed by a short inflationary period, to expand the photonic volume in order to low the temperature till the 


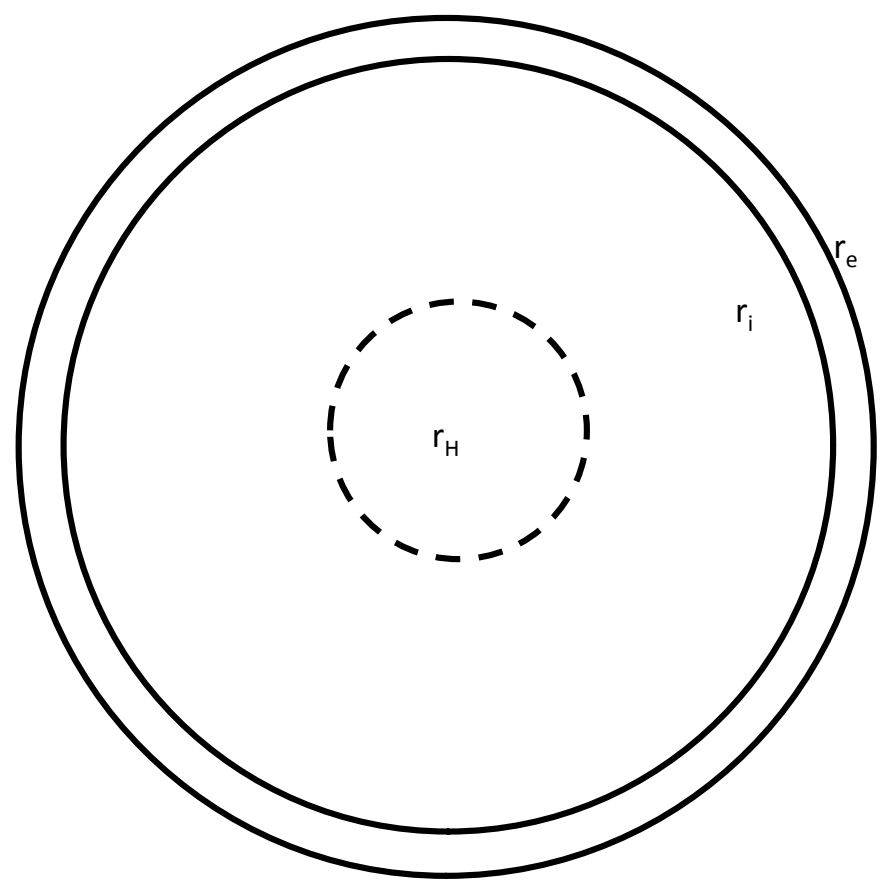

Figure 2. A cross section of the spherical Universe (not at scale); $r_{B}$ is the radius of the Big Bang $\sim 10^{17}(\mathrm{~cm}) ; r_{e}$ is the estimated radius of the Space $2 \times 10^{28}(\mathrm{~cm})$ and $r_{i}$ is the internal radius of the Physical Universe. The Observable Universe thickness is $\left(r_{e}-r_{i}\right) \sim 2 \times$ $10^{19}(\mathrm{~cm})$.

necessary level for matter condensation. Instead, at the end of the long Big Bang period proposed by reference [3], the photon gas temperature must have been in the order of $2 \times 10^{4} \mathrm{~K}$, adequate to permit the lepton's condensation. So, the Inflationary period should not be necessary [9].

\section{Some Consequences of the Big Bang}

In a previous work [1] it was assumed the Universe expansion as adiabatic since there is not any enter or loss of heat in the total Universe. It is important to point out several aspects already mentioned. The radial expansion velocity of the Big Bang is given by Equation (5); so, at the end of the Big Bang lifetime, such velocity was only $\sim 2 \times 10^{5}(\mathrm{~cm} / \mathrm{s})$. Anyway, the constancy of $c$ was kept by every one of the original photons across their continuous internal collisions. The Big Bang final radius would be $\boldsymbol{r}_{B} \sim 10^{17}(\mathrm{~cm})$ and the energy density would correspond to $5 \times 10^{45}\left(\mathrm{erg} / \mathrm{cm}^{3}\right)$. Consequently, the mean free path at the end of the Big Bang must have been too small to let the existence of a high fraction of low energy photons; these ones would increase and react in the subsequent period $\left(t_{c}\right.$ $\left.-t_{B}\right)$. Besides, accordingly to reference [10], the ratio of the present radial function to that of the Big Bang end implies a $z$ value $\sim 5 \times 10^{3}$.

Obviously it must have existed a mixture of frequencies and energies in the original photons which, later, devoted a small fraction $\left(\sim 10^{-20}\right)^{*}$ to condense a Physical Universe and, simultaneously, to loose energy by a redshift process and by collisions with the condensed matter, so producing the today known CMB 
spectrum [11]. Besides, the energy devoted to the Physical Universe creation could have generated the necessary number of Higgs photons (125 GeV/photon $\sim 0.2 \mathrm{erg} /$ photon) which corresponds to a temperature $\sim 10^{15}(\mathrm{~K})$, i.e., well inside the Big Bang.

In reference [1] it was applied the convective operator to the photon's energy to obtain the equation of continuity, which gives a luminosity, at the $t_{c}$ time, of $L$ $\sim 4 \times 10^{80}(\mathrm{erg} / \mathrm{s})$.

- The energy equivalent of the assumed mass $\left(3 \times 10^{58} \mathrm{~g}\right)$ of the Physical Universe is about $2.2 \times 10^{77}(\mathrm{erg})$.

\section{Some Necessary Metaphysical Speculations}

To date, the existence of the cosmic radiation background has been assumed as the best proof of a Big Bang origin of the Universe. Such conclusion has been adopted in this and a previous paper [1] that included, necessarily, several metaphysical concepts to try to explain some aspects of the creational task. So, the creation-ex-nihilo started with the Big Bang, whose characteristics have been summarized above, including the creation of time, space, matter and physical laws. Based on thermo-dynamical concepts of a photonic plasma, some steps have been mentioned as functions of temperature. Assuming that the Creator (or the creative force) had imposed himself an ethical condition, this one was transmitted to some of the created beings: those supplied with a conscience. So, the transcendence characteristic of the Creator was impressed in the eternal soul of the human beings; and his ethical quality was transmitted by means of the conscience and the free will. However, though the conscience would correspond to the soul, its ethical use depends on the human discernment, i. e. an intellectual capacity. The search for knowledge seems to be another characteristic of the human conscience (as a probable consequence of the original respect for the Tree of Life), a task without any time limit.

\section{Conclusions}

1) The relationship between the Hubble parameter and time has been given by Equation (5) as $H=\sqrt{2} / t\left(\mathrm{~s}^{-1}\right)$.

2) The Space expanding acceleration has been expressed as a constant by equation (4) as $\Gamma_{H}=2 \times 10^{-7}\left(\mathrm{~cm} / \mathrm{s}^{2}\right)$. Figure 1 shows the straight and the parabolic slants of the cones of time, which intersected at $t \sim 1.5 \times 10^{10}$ (y). So, the parabolic cone radius is today bigger than the previously assumed cone.

3) The positive Hubble potential of the Space expansion was deduced in Equation (7): $V_{H}=H^{2} r^{2} / 4$ as a growing function of the scale factor.

4) The time when the Space expansion reached the $c$ velocity, $t_{c}$ has been found to occur at $1 / 3$ of the present time $t_{o}$. Obviously, in the subsequent period $\left(t_{o}-t_{c}\right)$, the total Space expanded at higher velocities than $c$, carrying together the Physical Universe; though, inside this one, the $c$ value of matter remained as a constant. Besides, after the $t_{c}$ time, it had been deduced the possibility of an 
imaginary time domain [7] that would be the characteristic coordinate in an Euclidic Space [8]. Assuming that the present radial function of the Physical Universe would correspond to the most distant object detected, with $z \sim 12$; and also assuming that after the $t_{c}$ time the most external objects are traveling at higher velocities than $c$, it could be necessary to apply similar equations to the above named (2 - 14). Otherwise, the confirmation of the Physical Universe expansion must have probably been based on an unexpected speed of the $(z \sim 12)$ objects; so this would be, to date, the limit of the Physical Universe. Assuming that such observation was made from the middle of the spherical shell, it means a thickness of this shell of $\boldsymbol{r}_{p u}<2 \times 10^{19}(\mathrm{~cm})$ as shown in Figure 2 for the Physical or Visible Universe.

5) The condensation of photons in leptons could only have occurred at the end of the Big Bang duration ( 32,000 y) when the temperature was lower than $2 \times 10^{4}(\mathrm{~K})$, i.e. the binding energy of leptons. After, they continued the synthesis processes of quarks, atoms and stars as well as manifestations of the different types of forces, to integrate the present Physical Universe. These conclusions imply that the generally assumed inflationary period, following a short Big Bang, should not be necessary. Furthermore, for simplicity, it has been supposed that the creation of original photons stopped at the Big Bang end. So, because of the $c$ constancy inside the Physical Universe, this has acquired a ring geometry (Figure 2): so, a small fraction of photons started a mass condensation for the Physical Universe though, most of them, suffered a redshift process till the present CMB spectrum. Therefore, the mass generation would have occurred between the $\left(t_{c}-t_{B}\right)$ period, by nuclear reactions and later, by electro-magnetic and gravitational forces.

6) Related to metaphysical propositions (already discussed), it has been concluded that the creation ex-nihlo started with the Big Bang, together the time, Space, matter and physical laws. The first law could have been the expansive acceleration of Space and, consequently, that of matter. Besides, the transcendence and ethical characteristics of the Creator were transmitted to the human kind by means, respectively, of an eternal soul and a conscience (with a free will). The eternal search for knowledge seems also to have been impressed in the human conscience.

\section{Conflicts of Interest}

The author declares no conflicts of interest regarding the publication of this paper.

\section{References}

[1] Lartigue, J.G. (2018) Journal of Modern Physics, 9, 2443-2456.

[2] Lartigue, J.G. (2019) Applied Physics Research, 11, 70. https://doi.org/10.5539/apr.v11n4p70

[3] Hogan, C.J. (1999) Energy Flow in the Universe.

[4] Leff, H.S. (2002) American Journal of Physics, 70, 795. 
https://doi.org/10.1119/1.1479743

[5] International Astronomical Union (2018) Resolution B4.

[6] Riess, A., et al. (1998) Astronomical Journal, 116, 1009-1038. https://doi.org/10.1086/300499

[7] Lartigue, J.G. (2020) Applied Physics Research. (In Press)

[8] Hawking, S. (1988) Historia del Tiempo. Ed. Crítica, México, 179.

[9] Hossenfelder, S. (2017) Is the Inflationary Universe a Scientific Theory? Not Anymore. https://www.forbes.com/sites/startswithabang/2017/\#77d4d994370a

[10] Sartory, L. (1996) Understanding Relativity. UCLA, 303.

[11] Sartory, L. (op. cit.) Figure 9.18. 